\title{
PARTICLE ACCELERATION IN THE HELIOSPHERE
}

\author{
LOUKAS VLAHOS \\ Department of Physics \\ University of Thessaloniki, \\ 54006 Thessaloniki, GREECE
}

The heliosphere could be divided in three major acceleration Laboratories, the solar surface (Laboratory 1), the interplanetary medium (Laboratory 2) and Earth and Planetary magnetospheres (Laboratory 3). Our understanding of the acceleration process depends strongly on the nature of the drivers and the energy dissipation process. The energy gain by a particle with velocity $\vec{v}$ is $\Delta E(t)=\int \vec{E}(\vec{r}, t) \cdot \vec{v}(\vec{r}, t) d \vec{r}$, where $\vec{E}(\vec{r}, t)$ is the variation of the electric field in space and time. All three Laboratories mentioned above share a common characteristic, the drivers and the energy dissipation processes are closely connected to fully developed MHD turbulence. We can show that our understanding of particle acceleration depends strongly on the interaction of particles with fields resulting from fully developed MHD turbulence.

The following processes have been used for the study of particle acceleration in the heliosphere: (1) Neutral sheets and turbulent neutral sheets, (2) Shock and turbulent shocks, (3) Double layer $E$-field and randomly distributed $E$-fields, (4) Small amplitude MHD waves. It is obvious that the turbulent nature of the fields has been added to neutral sheets, shocks, $E$-fields to enhance the efficiency of the acceleration process.

The standard diffusion equation used for particle acceleration process is

$$
\frac{\partial f}{\partial t}=\frac{\partial}{\partial p} D_{\mathrm{pp}} \frac{\partial f}{\partial p}
$$

where $f$ is the velocity distribution function, $p$ is the momentum and $D_{\mathrm{pp}}$ is the diffusion coefficient in the momentum space

$$
D_{\mathrm{pp}} \sim(\delta B / B)^{2}
$$

where $(\delta B / B)$ are the field fluctuations. Eq. (1) is applicable only when $(\delta B / B)$ are much smaller than one. 
It is obvious that Eq. (1) cannot handle fluctuation when $(\delta B / B)>>1$. Current theories on fully developed MHD turbulence have emphasised the role played by strong intermittent structures where $(\delta B / B)>1$ inside the turbulent flow. These coherent nonlinear structures play a major role on particle acceleration. The diffusion coefficient in such a turbulent environment is very different from the one shown in Eq. (2). Inside fully developed turbulence, coherent structures like neutral sheets, shocks, randomly placed $E$-fields appear naturally. A new generation of particle acceleration mechanisms will be developed in the near future where a collection of structures will appear inside the turbulent flow.

We outline the approach used for particle acceleration in the Laboratories mentioned above:

- The study of particle acceleration inside the solar atmosphere is based on the non-linear fields developed during (1) the formation and evolution of active regions, (2) the formation and interaction of magnetic discontinuities, (3) MHD waves inside magnetic discontinuities and a collection of strong $E$-fields.

The driver for the formation and evolution of an active region is the turbulent convection zone. A spectrum of magnetic flux tubes raised above the solar surface. A part of the flux tube is still inside the convection zone. The turbulent convection zone forms stochastic magnetic fields inside the active region. A large number of neutral sheets and shock waves are spontaneously formed. The study of the energy gain by electrons and protons inside a complex active region driven by the turbulent convection zone has produced a number of interesting results.

- In the Interplanetary medium, the driver is the fast hydrodynamic flow. Formation of a series of interplanetary shocks and the turbulent fields around the shock play a crucial role on particle acceleration. Stochastic magnetic field lines return many times in the surface of the shock and dramatically enhance the shock-particle interaction.

- In the rotating Magnetosphere the driver is the turbulent solar wind. Several unique structures are formed (1) the turbulent Bow shock, (2) Random $E$-fields inside the Aurora, (3) Radiation belts and the (4) turbulent neutral sheets at the tail. Here again a collection of non-linear discontinuities accelerate the particles.

Our main points are:

- Turbulent motion dominates the acceleration of particles in the heliosphere. Organised forms inside fully developed turbulence cause many energetic phenomena, e.g. flares, interplanetary shocks, Auroras, Bow shocks, magnetospheric tail etc. 
- Structures like shocks, neutral sheets, double layers, large amplitude waves propagating inside turbulent media are common in the heliosphere.

- These structures have an intermittent behaviour and are part of the energy release process.

- The new generation of accelerators will include a variety of non-linear structures e.g. ensembles of shocks, neutral sheets, collection of double layers and waves, so we believe that the developments and understanding of MHD turbulence will go hand by hand with the development of new acceleration processes in the heliosphere. 